\title{
Weakly stable state of the crystal lattice of metals and alloys
}

\author{
S. V. Makarov ${ }^{1}$, V. A. Plotnikov ${ }^{1, \dagger}$, B. F. Dem'yanov², A. I. Potekaev ${ }^{3}$ \\ †plotnikov@phys.asu.ru \\ ${ }^{1}$ Altai State University, 61 Lenina Av., Barnaul, 656049, Russia \\ ${ }^{2}$ Altai State Technical University n. a. I. I. Polzunov, 46 Lenina Av., Barnaul, 656038, Russia \\ ${ }^{3}$ Siberian Physical-Technical Institute, 1 Novosobornaya Sq., Tomsk, 634050, Russia
}

The state of the crystalline medium under the conditions of thermal fluctuations and mechanical stresses called weakly stable is determined by the displacement of atoms at a relative distance of $0.15-0.20$ from the equilibrium positions. Such a displacement of the atoms follows the Lindemann criteria of the crystals melting, and the state of atomic ensemble can be described as delocalized one. The state of delocalization means the transition of the atom through the maximum of interaction force and is characterized by a strong anharmonic component of oscillations and softening of elastic modules. It is the state of delocalization of the atomic ensemble that is the weakly stable state of the crystal lattice. Weakly stable state of metal materials is manifested in a wide range of phenomena. These include abnormal reduction of elastic modules in thermoelastic martensitic transformations, second-order structural-phase transitions with the formation of long-period structures, abrupt plastic deformation, most clearly manifested in aluminium and its alloys, increasing the rate of deformation during creep in the ultrasonic field under static load. This paper presents the results of the analysis of the weakly stable state of the crystalline medium, especially in plastic deformation, that is, in the processes caused by the displacement of atoms from the equilibrium position. In these processes, the weakly stable state of the system can occur in the field of mechanical stresses and thermal fluctuations when the acoustic field of standing waves is applied, formed by primary acoustic emission signals, the combined action of which allows to overcome the potential barrier. In this state, the oscillatory displacement of the acoustic standing wave acts as a factor of self-organization, that is, it may be sufficient to activate the correlated dislocation slip, in fact, the athermal above-barrier slip in the weakly stable state of the crystal lattice.

Keywords: weakly stable state, acoustic emission, acoustic correlation of elementary deformation acts, Lindemann criterion, delocalization of atoms.

\section{Слабоустойчивое состояние кристаллической решетки металлов и сплавов}

\author{
Макаров С. В. ${ }^{1}$, Плотников В. А. ${ }^{1, \dagger}$, Демьянов Б. Ф. ${ }^{2}$, Потекаев А. И. ${ }^{3}$ \\ †plotnikov@phys.asu.ru
}

\begin{abstract}
${ }^{1}$ Алтайский государственный университет, пр. Ленина, 61, Барнаул, 656049, Россия
${ }^{2}$ Алтайский государственный технический университет им. И. И. Ползунова, пр. Ленина, 46, Барнаул, 656038, Россия

${ }^{3}$ Сибирский физико-технический институт, пл. Новособорная, 1, Томск, 634050, Россия
\end{abstract}

Состояние кристаллической среды, находящейся в условиях тепловых флуктуаций и механических напряжений, именуемое слабоустойчивым, определяется смещением атомов на относительное расстояния $0.15-0.20$ от положения равновесия. Такое смещение атомов отвечает критерию плавления кристаллов Линдемана, а состояние атомного ансамбля можно характеризовать как делокализованное. Состояние делокализации означает переход атома через максимум силы взаимодействия и характеризуется сильной ангармонической составляющей колебаний и размягчением упругих модулей. Именно состояние делокализации атомного ансамбля и является слабоустойчивым состоянием кристаллической решетки. Слабоустойчивое состояние металлических материалов проявляется в широком круге явлений. К ним относятся аномальное снижение упругих модулей при термоупругих мартенситных превраще- 
ниях, структурно-фазовые переходы второго рода с образованием длиннопериодических структур, скачкообразная пластическая деформация, наиболее ярко проявляющаяся в алюминии и его сплавах, увеличение скорости деформации в ходе ползучести в ультразвуковом поле при статической нагрузке. В данной работе представлены результаты анализа слабоустойчивого состояния кристаллической среды, прежде всего, при пластической деформации, то есть в процессах, обусловленных смещением атомов из положения равновесия. В этих процессах слабоустойчивое состояние системы может возникнуть в поле механических напряжений и тепловых флуктуаций при наложении акустического поля стоячих волн, сформированных первичными сигналами акустической эмиссии, совместное действие которых позволяет преодолеть потенциальный барьер процесса. В таком состоянии колебательное смещение акустической стоячей волны выступает фактором самоорганизации, то есть может быть достаточным для активации коррелированного дислокационного скольжения, фактически атермического надбарьерного скольжения в слабоустойчивом состоянии кристаллической решетки.

Ключевые слова: слабоустойчивое состояние, акустическая эмиссия, акустическая корреляция элементарных деформационных актов, критерий Линдемана, делокализация атомов.

\section{1. Введение}

Слабоустойчивое состояние металлических материалов, претерпевающих, например, термоупругие мартенситные превращения, традиционно связывают с аномально низкими упругими модулями [1]. Например, в никелиде титана аномальное снижение упругих модулей обусловлено смягчением фононных мод вблизи превращения В2 $\rightarrow$ B19' $[1,2]$. Наличие мягких сдвиговых мод $\{110\}<110>$ приводит к сдвиговой неустойчивости решетки В2-фазы и снижению напряжения мартенситного сдвига. Так в сплаве Au-47.5 ат.\% Cd напряжение мартенситного сдвига при достижении температуры начала превращения снижается фактически до нуля $[1,3]$. Слабоустойчивое состояние характерно и для сплавов, испытывающих структурно-фазовые переходы второго и близкие ко второму рода, где слабоустойчивость обусловлена длиннопериодическими состояниями вблизи границы потери устойчивости $[4,5]$. При этом кооперативная перестройка кристаллической решетки В2-фазы в ходе B2 $\rightarrow$ B19' превращения не требует разрыва межатомных связей.

Однако существует широкий круг процессов, для реализации которых необходим разрыв межатомных связей: процессы диффузии, трещинообразования, пластической деформации и другие. В этих процессах особое состояние системы, именуемое слабоустойчивым, может быть связано с состоянием атомного ансамбля в поле механических напряжений и тепловых флуктуаций, совместное действие которых позволяет преодолеть потенциальный барьер процесса [6,7]. Моделирование таких флуктуаций в атомной системе позволило установить, что сильная флуктуация энергии атомов может представлять собой достаточно устойчивое, динамическое состояние [8]. Особенностью тепловых колебаний атомов являются «динамические коллективные (кооперативные) атомные смещения», представляющие собой упорядоченные смещения групп атомов [9]. Внешнее механическое нагружение металлов, таких как медь, никель, алюминий, сопровождается увеличением концентрации динамических коллективных смещений и изменением энтальпии активации диффузионных процессов [6-10]. Таким образом, слабоустойчивое состояние можно связать со смещением атомов из положений равновесия, то есть с выходом атомного ансамбля из состояния равновесия и может характеризоваться как метастабильное.
В условиях воздействия механического напряжения и температуры среднее время ожидания элементарного акта разрыва связи зависит от эффективной величины потенциального барьера, преодоление которого осуществляется термофлуктуационным путем $[7,11,12]$. При этом эффективный порог активации может значительно снижаться фактически до нуля, характеризуя особое состояние атомного ансамбля. В таком состоянии кристаллической решетки пластическое течение связано со слабоустойчивым состоянием, включающем и локальную потерю сдвиговой устойчивости в зоне действия концентраторов напряжений $[3,6]$, когда движение дислокационного сегмента осуществляется надбарьерно атермическим путем.

В настоящее время считается очевидным, что пластическое течение, особенно большие пластические деформации, не могут быть описаны как аддитивный вклад отдельных дислокаций в накопление деформации [13]. Эксперименты по деформированию металлов свидетельствуют [14], что в ходе пластической деформации в направлении растяжения распространяются очаги деформации, в которых локализовано пластическое течение. Локализация пластического течения кристаллической структуры при пластической деформации для классической трехстадийной зависимости «механическое напряжение - относительная деформация» обычно связывается с процессом самоорганизации дислокаций, проявляющейся в формировании линий и полос скольжения $[15,16]$. Это может свидетельствовать о возникновении локальной слабой устойчивости (или неустойчивости) атомной системы в условиях термомеханического нагружения [17], своеобразно (в виде иерархически взаимосвязанных коррелированных событий) проявляющейся на разных стадиях пластической деформации.

Сопровождающая эволюцию структуры акустическая эмиссия отражает процессы самоорганизации, характеризует слабую устойчивость в атомной подсистеме при внешнем воздействии, а скачкообразная деформация и связанные с ней акустические импульсы свидетельствуют о пространственно-временной упорядоченности движения дефектов [18]. Обнаруженные при высокотемпературной деформации алюминия $[19,20]$ макроскопические деформационные скачки и аномально высокоамплитудные сигналы акустической эмиссии следует проанализировать с позиций слабой устойчивости (или потери устойчивости) кристалли- 
ческой решетки и корреляции элементарных деформационных актов в области локализации пластического течения. Характерно, что все события, связанные с пластической деформацией, распространением акустических волн и т.д., сопровождаются смещениями атомов из положения равновесия.

\section{2. Анализ экспериментальных ситуаций}

\section{1. Скачкообразная дебормачия}

Наиболее типичным явлением, связанным со смещением атомов из положения равновесия в условиях механического нагружения, является пластическая деформация, которая в широком интервале температур может представлять собой совокупность скачкообразных и монотонных актов накопления деформации [21-24].

Скачкообразная деформация, наиболее изученная в алюминии и его сплавах [23,25], проявляется в виде зубцов, возникающих на зависимости напряжение-деформация как результат релаксации напряжений и существенно зависит от температуры $[26,27]$. В алюминиево-магниевых сплавах скачкообразная деформация протекает как прерывистая текучесть и сопровождается акустической эмиссией $[28,29]$.

Эффект скачкообразной деформации относится к наиболее значимым проявлениям самоорганизации в ходе пластической деформации в металлах и сплавах [15]. Учет фактора самоорганизации позволяет сформулировать фундаментальный вывод о том, что наблюдаемое в экспериментах многообразие деформационного поведения и дислокационных структур есть результат эволюции дислокационного ансамбля путем развития коллективных и кооперативных явлений и их пространственной $[30,31]$ и временной (наше добавление) [25] упорядоченности, проявляющейся в формировании линий и полос скольжения.

Однако существенно то, что проявление самоорганизации в системе дислокаций в ходе пластической деформации обсуждается без анализа обратных связей. То есть в самоорганизующейся системе линейных дефектов вне обсуждения оказывается фактор самоорганизации.

\section{2. Дебормация в ультразвуковом поле}

Смещения атомов в кристалле могут быть вызваны не только статическим силовым воздействием, но и динамическим воздействием ультразвуковых колебаний, возбуждаемых в металлах и сплавах. Ускорение ползучести металлов в ультразвуковом поле, возбуждаемом на частоте 20 кГц, отмечено во многих работах [32 - 35].

Увеличение скорости накопления деформации в ультразвуковом поле и в условиях действия статической нагрузки может быть обусловлено слабоустойчивостью кристаллической решетки исследуемых алюминия или меди и не является простым суммированием статического напряжения и амплитуды ультразвуковых колебаний $[35,36]$. Это состояние особенно заметно в области концентрации колебательной энергии ультразвука в пучности стоячей продольной волны $[28,29]$.

\section{3. Пластическая деформация и акустическая эмиссия}

Очевидно, смещения атомов из положения равновесия могут быть вызваны акустическими волновыми пакетами, обусловленными акустической эмиссией в процессе пластической деформации. Более того такие смещения в силу волнового характера являются коррелированными смещениями, а в случае стоячих акустических волн такие смещения могут быть охарактеризованы как кооперативные [25, $34-38]$.

Попытки использовать акустическую эмиссию для исследования природы скачкообразной деформации продолжаются до сих пор [23]. Корреляция между движением дислокаций и импульсами акустической эмиссии трактуется таким образом, что акустические всплески вызываются лавинообразным прохождением дислокаций. Однако по-прежнему акустическая эмиссия выступает в роли сопутствующего явления. Воздействие самих акустических колебаний на процесс движения дислокаций не рассматривается.

В то же время в наших работах показано $[25,37-38]$, что амплитуда акустического сигнала (точнее квадрат амплитуды) может служить мерой корреляции элементарных деформационных актов в связи с тем, что выход на поверхность системы дислокационных сегментов одной системы скольжения генерирует систему когерентных акустических волн, интерференция которых формирует единичный акустический сигнал аномально большой амплитуды. Кроме того установлено [38], что в деформируемом объеме формируется система стоячих акустических низкочастотных волн, в которых запасается заметная по величине колебательная энергия акустической эмиссии. В этой связи можно предполагать, что сигналы акустической эмиссии, трансформированные в низкочастотные акустические стоячие волны, осуществляют активацию и корреляцию элементарных деформационных актов, то есть выполняют роль фактора самоорганизации в макроскопическом объеме в слабоустойчивой кристаллической среде.

В данной работе представлены результаты анализа слабоустойчивого состояния кристаллической среды, прежде всего, при пластической деформации, то есть в процессах, обусловленных смещением атомов из положения равновесия.

\section{3. Слабоустойчивое состояние кристаллической решетки и ангармонизм межатомного взаимодействия}

Будем полагать, что слабоустойчивое состояние кристаллической решетки связано с нелинейностью межатомного взаимодействия. Для анализа нелинейных эффектов можно использовать парный межатомный потенциал Морзе, построенный для алюминия по методике, описанный ранее [39]

$$
\varphi_{K L}=D_{K L} \beta_{K L}\left[\beta_{K L} \exp \left(-2 \alpha_{K L}\left|\vec{r}_{i}-\vec{r}_{j}\right|\right)-2 \exp \left(-\alpha_{K L}\left|\vec{r}_{i}-\vec{r}_{j}\right|\right)\right],
$$


где $\varphi_{K L}-$ потенциал межатомного взаимодействия атомов сорта $K, L, \vec{r}_{i}$ и $\vec{r}_{j}$ - радиус-векторы атомов $i$ и $j$, соответственно, $D_{K L}, \alpha_{K L}, \beta_{K L}$ - параметры потенциала межатомного взаимодействия между атомами сорта $K$ и $L$, значения которых для алюминия и меди приведены в Табл. 1.

Данный потенциал был апробирован при расчетах структуры и энергии границ зерен различного типа и процессов зернограничной самодиффузии и показал хорошее согласие рассчитанных свойств с экспериментальными значениями $[40,41]$.

Параметры потенциала Морзе определяли из набора экспериментальных данных по энергии сублимации, параметру решётки и объёмному модулю упругости (Табл. 2).

Зависимость парной потенциальной функции Морзе $\varphi(r)$ для идеальной кристаллической решётки алюминия от межатомного расстояния, построенная с использованием параметров $D_{K L}, \alpha_{K L}, \beta_{K L}$, приведена на Рис. 1 a. На Рис. $1 \mathrm{~b}$ приведена также и зависимость модуля силы межатомного взаимодействия от положения атома. Несмотря на то, что взаимодействие рассмотрено для двух атомов, потенциал Морзе учитывает соседство в пяти координационных сферах. Поэтому полученные численные оценки соответствуют поведению атомов в кристалле алюминия. При смещении атома из положения равновесия сила изменяется сложным образом. Линейная зависимость силы, соответствующая закону Гука, существует только в узком интервале смещений вблизи положения равновесия, при больших смещениях линейность существенно нарушается.

На графиках потенциала и силы межатомного взаимодействия можно выделить три особые точки положения атомов алюминия. Точка $1\left(r_{0}=1.118 \AA\right)$ соответствует равновесному положению атомов в решетке, результирующая сила, действующая со стороны соседних атомов, в этом положении равна нулю. Смещения атома из положения равновесия, как при сжатии, так и при растяжении приводит к линейному возрастанию силы. Это соответствует линейной области упругой деформации. Точка 2 (отклонение от положения равновесия равно $\Delta r_{e}=0.07 \AA$ ) показывает величину смещения, при превышении которой линейный характер зависимости между силой и деформацией нарушается. Это область нелинейной деформации. Точка 3 соответствует точке перегиба зависимости потенциала $\varphi(r)$, в которой силовая характеристика равна максимальному значению.

При отклонении атома на расстояние большее $\Delta r_{m}=0.234 \AA$ сила взаимодействия начинает уменьшаться. Эта точка, соответствующая максимальному значению силы действующей на атом при растяжении кристалла, является своего рода силовым барьером. Смещение атомов до величины $\Delta r_{m}$ приводит их в состояние делокализации. То есть, делокализацию атомов при смещении их до величины $\Delta r_{m}$, можно трактовать как появление слабоустойчивого состояния кристалла. Кроме того такое состояние атомной системы характеризуется сильным ангармонизмом колебательного движения атомов.

Действительно, проведенные расчеты свидетельствуют, что область линейной зависимости
Табл. 1. Значения параметров $D_{K L}, \alpha_{K L}, \beta_{K L}$ потенциала межатомного взаимодействия для алюминия и меди.

Table 1. Values of parameters $D_{K L}, \alpha_{K L}, \beta_{K L}$ of interatomic interaction potential for aluminium and copper.

\begin{tabular}{|c|c|c|c|}
\hline Металл/metal & $\alpha_{K L}, \AA^{-1}$ & $\beta_{K L}$ & $D_{K L}$, эB/eV \\
\hline Алюминий/Aluminum & 2.959 & 27.368 & 0.3176 \\
\hline Медь/Copper & 3.9278 & 41.598 & 0.3736 \\
\hline
\end{tabular}

Табл. 2. Экспериментальные значения физических величин для определения параметров потенциала Морзе.

Table. 2. Experimental values of physical quantities to determine the parameters of the Morse potential.

\begin{tabular}{|c|c|c|c|}
\hline $\begin{array}{c}\text { Металл } \\
\text { Metal }\end{array}$ & $\begin{array}{c}\text { Энергия } \\
\text { сублимации, } \\
\text { кдж/моль } \\
\text { Energy of } \\
\text { sublimation, } \\
\mathrm{kJ} / \mathrm{mol}\end{array}$ & $\begin{array}{c}\text { Пешётки, нм } \\
\text { Lattice } \\
\text { parameter, } \\
\mathrm{nm}\end{array}$ & $\begin{array}{c}\text { Объёмный модуль } \\
\text { упругости, ГПа } \\
\text { ulasticity, GPa }\end{array}$ \\
\hline $\begin{array}{c}\text { Алюминий } \\
\text { Аluminium }\end{array}$ & 230 & 0.40403 & 75.8 \\
\hline $\begin{array}{c}\text { Медь } \\
\text { Соррег }\end{array}$ & 340 & 0.36150 & 137.6 \\
\hline
\end{tabular}
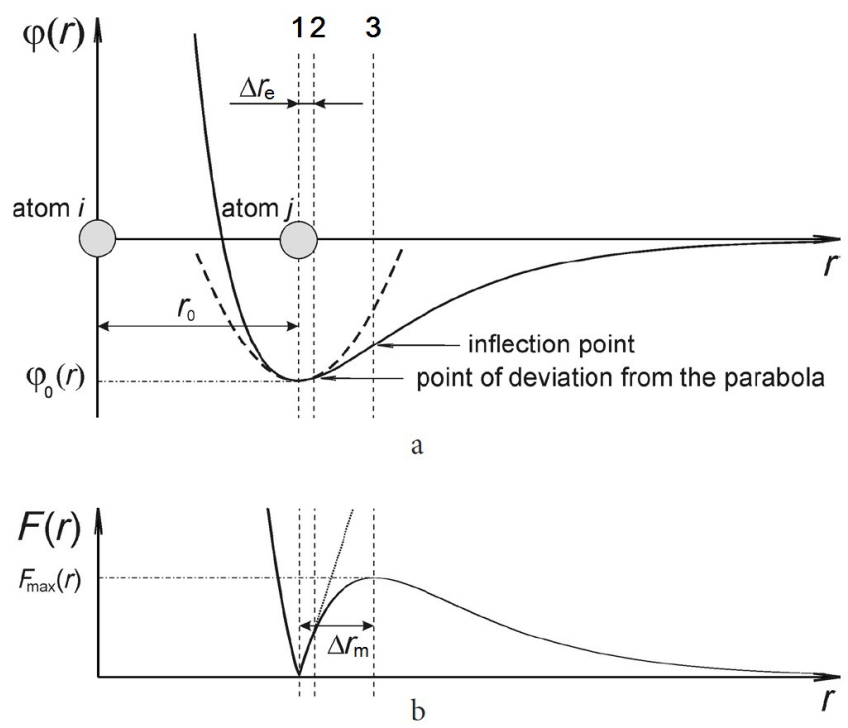

Pис. 1. Потенциал межатомного взаимодействия в идеальном кристалле алюминия (a), сила межатомного взаимодействия (b).

Fig. 1. The interatomic potential in a perfect crystal of aluminium (a), the interatomic force (b).

силы от величины смещения составляет всего 4-5\% от межатомного расстояния, а смещение атомов, соответствующее максимуму силы и равное примерно 20\% параметра решетки, удовлетворяет критерию плавления кристаллов Линдемана [42]. Согласно этому критерию при температуре плавления кристаллов среднеквадратичная амплитуда тепловых колебаний атомов достигает относительной величины $\delta=0.15-0.20$ межатомного расстояния, что составляет заметную 
долю этого расстояния для разных кристаллов [43]. То есть в случае плавления состояние кристалла может соответствовать делокализованному положению атомов [44] - положению атомов в точке 3. Делокализованное состояние атомов (или слабоустойчивое состояние в наших терминах) кристаллической решётки [43] можно рассматривать как возбужденное, а положение атома соответствует состоянию в промежуточной (метастабильной) потенциальной яме [45]. Иными словами слабоустойчивое состояние представляет собой особое состояние, отделенное от основного состояния небольшим потенциальным барьером.

Таким образом, смещения атомов от положения равновесия, как динамические (при нагреве), так и статические (в условиях действия механических напряжений) приводят к потере устойчивости решетки вследствие уменьшения силы межатомного взаимодействия и связанного с этим увеличения амплитуды колебаний атомов. Возникновение таких аномальных эффектов обусловлено сильным ангармонизмом и размягчением упругих модулей, что сопровождается изменением фононного спектра в кристалле, например, в условиях сжатия [46]. Упругие модули в условиях сжатия оказываются чувствительны к конфигурации напряженного состояния, то есть уменьшение модуля $C^{\prime}=1 / 2\left(C_{11}-C_{12}\right)$ в нагруженном кристалле приводит к механической неустойчивости решетки. Эти аномалии особенно заметны в окрестностях дефектов, служащих центрами зарождения пластической деформации.

\section{4. Заключение}

Проведенный анализ состояния кристаллической решетки, именуемого слабоустойчивым, свидетельствует о переходе атомного ансамбля в поле механических напряжений в делокализованное состояние, характеризуемое $20 \%$ смещением атомов из положения равновесия и увеличением амплитуды колебаний атомов, то есть переходе в возбужденное (неравновесное) состояние. Возрастание амплитуды колебаний атомов сопровождается усилением ангармонизма и размягчением упругих модулей, что сопровождается изменением фононного спектра в кристалле.

Очевидно, внешнее механическое воздействие (поле напряжений), локализованное на дефектах, переводящее кристаллическую среду в состояние слабой устойчивости, снижает эффективный порог активации разрыва связи. В таком состоянии колебательное смещение акустической стоячей волны, сформированное первичными сигналами акустической эмиссии, выступает фактором самоорганизации, то есть может быть достаточным для активации коррелированного дислокационного скольжения, фактически атермического надбарьерного скольжения в слабоустойчивом состоянии кристаллической решетки.

Иными словами, в уравнении Журкова для времени ожидания разрыва связи следует учитывать не только работу статических сил, но еще и работу динамических сил (фактически именно этот эффект обуславливает повышение скорости ползучести в ультразвуковом поле).
Таким образом, уменьшение эффективного порога активации за счет работы статических сил, локализованных на структурном элементе, и также за счет работы динамических сил акустического поля стоячих волн, сформированных первичными сигналами акустической эмиссии, повышает вероятность разрыва межатомной связи тепловыми флуктуациями.

\section{Литература/References}

1. I. Nakanishi. Softening of the lattice and the nature EMF. The shape memory effect in alloys. Moscow, Metallurgiya (1979) 155 p. (in Russian) [И. Никаниши. Смягчение решетки и природа ЭЗФ. Эффект памяти формы в сплавах. Москва, Металлургия (1979) 155 с.]

2. A.I. Potekaev, A.A. Klopotov, E. V. Kozlov, V.V. Kulagina. Pretransitional weakly stable structures in nickelide titanium. Tomsk, NTL (2004) 296 p. (in Russian) [А.И. Потекаев, А.А. Клопотов, Э.В. Козлов, В.В. Кулагина. Слабоустойчивые предпереходные структуры в никелиде титана. Томск, НТЛ (2004) 296 с.]

3. K. Mukherjee, M. Chandrasekaran, F. Millo. Transformation of the pre-martensitic phase into martensitic associated with the shape memorization effect. The shape memory effect in alloys. Moscow, Metallurgiya (1979) p. 128-171. (in Russian) [К. Мукердж, M. Чандрэсикэрэн, Ф. Милилло. Превращение предмартенситной фазы в мартенсит, связанное с эффектом запоминания формы. Эффект памяти формы в сплавах. Москва, Металлургия (1979) с. 128 -171.]

4. A. I. Potekaev, S. V. Dmitriev, V.V. Kulagin et al. Weakly stable long-period structures in metal systems. Tomsk, NTL (2010) 308 p. (in Russian) [А.И. Потекаев, С. В. Дмитриев, В. В. Кулагина и др. Слабоустойчивые длиннопериодические структуры в металлических системах. Томск, НТЛ (2010) 308 с.]

5. A.I. Potekaev, M.D. Starostenkov, V.V. Kulagina. Influence of point and planar defects on structural-phase transformations in the pre-transition weakly stable region of metal systems. Tomsk, NTL (2014) 488 p. (in Russian) [А.И. Потекаев, М.Д. Старостенков, В.В. Кулагина. Влияние точечных и планарных дефектов на структурно-фазовые превращения в предпереходной слабоустойчивой области металлических систем. Томск, НТЛ (2014) 488 с.]

6. S. V. Makarov, V.A. Plotnikov, A.I. Potekaev. Russian Physics Journal. 54 (3), 314 (2011).

7. V.R. Regel, A.I. Slutsker, E.V. Tomashevsky. Kinetic nature of strength of solids. Moscow, Nauka (1974) 560 p. (in Russian) [В.Р. Регель, А.И. Слуцкер, Э.В. Томашевский. Кинетическая природа прочности твердых тел. Москва, Наука (1974) 560 с.]

8. A. I. Slutsker, A. I. Mihailin, I. A. Slutsker. Phys.-Usp. 37, 335 (1994). DOI: 10.1070/PU1994v037n04ABEH000017

9. G. M. Poletaev, M.D. Starostenkov. Physics of the Solid State, 51 (4) 686 (2009). (in Russian) [Г.М. Полетаев, М. Д. Старостенков. ФТТ, 51 (4) 686 (2009).]

10. M.S. Feldman, G. M. Poletaev, R. Y. Rakitin, M.D. Star ostenkov. Fundamental problems of modern materials science. 2 (1), 64 (2005) (in Russian) [M. С. Аксенов, 
Г.М. Полетаев, Р.Ю. Ракитин, М.Д. Старостенков. Фундаментальные проблемы современного материаловедения. 2 (1), 64 (2005).]

11. A. I. Slutsker. Physics of the Solid State. 46 (9) 1606 (2004) (in Russian) [А.И. Слуцкер. ФТТ. 46 (9) 1606 (2004).]

12. M.M. Myshlyaev. Creep of polygonized structures. Imperfections of the crystal structure and martensitic transformations. Moscow, Nauka (1972), pp. 194-234. (in Russian) [M. М. Мышляев. Ползучесть полигонизованных структур. Сб. Несовершенства кристаллического строения и мартенситные превращения. Москва, Наука (1972), с. $194-234$.

13. V.V. Rybin. Large plastic deformation and fracture of metals. Moscow, Metallurgiya (1986), 224 p. (in Russian) [В.В. Рыбин Большие пластические деформации и разрушение металлов. Москва, Металлургия (1986) 224 с.]

14. L.B. Zuev, V.I. Danilov, V.V. Gorbatenko. Technical Physics. 65 (5) 91 (1995). (in Russian) [Л.Б. Зуев, В. И. Данилов, В. В. Горбатенко. ЖТФ. 65 (5) 91 (1995).]

15. G. A. Malygin. Phys.-Usp., 42887 (1999).

16. V.E. Panin, V.A. Likhachev, Yu. V. Grinyaev. Structural levels of deformation of solids. Novosibirsk, Nauka (1985) 230 p. (in Russian) [В.Е. Панин, В.А. Лихачев, Ю.В. Гриняев. Структурные уровни деформации твердых тел. Новосибирск, Наука (1985) 230 с.]

17. N. A. Koneva, L. I. Trishkina, A. I. Potekaev, E. V. Kozlov. Structural-phase transformations in weakly stable states of metallic systems under thermal and force effects. Tomsk, NTL (2015) 344 p. (in Russian) [Н. А. Конева, Л.И. Тришкина, А.И. Потекаев, Э.В. Козлов. Структурно-фазовые превращения в слабоустойчивых состояниях металлических систем при термосиловом воздействии. Томск, НТЛ (2015) 344 с.]

18. V.N. Bovenko. Bulletin of the Academy of Sciences of the USSR. Metals. 1, 129 (1984). (in Russian) [В. Н. Бовенко. Изв. АН СССР. Металлы. 1, 129 (1984).]

19. V.A. Plotnikov V.A., Makarov. Russian Physics Journal. 48 (11), 1142 (2005).

20. V.A. Plotnikov, S. V. Makarov. Deformation and destruction of materials. 3, 27 (2005). (in Russian) [В. А. Плотников, С. В. Макаров. Деформация и разрушение материалов. 3, 27 (2005).]

21. V. P. Lebedev, V.S. Krylov, S. V. Lebedev, S. V. Savic. Physics of the Solid State. 49 (11), 1994 (2007). (in Russian) [В.П. Лебедев, В.С. Крыловский, С.В. Лебедев, С. В. Савич. ФТТ. 49 (11), 1994 (2007).]

22. N.N. Peschanskaya, V. V., Shpeisman, A.B. Sinani, B. I. Smirnov. Physics of the Solid State. 46 (11), 1991 (2004). (in Russian) [Н. Н. Песчанская, В. В. Шпейзман, А. Б. Синани, Б. И. Смирнов. ФТТ. 46 (11), 1991 (2004).]

23. M.A. Lebyodkin, I.V. Shashkov, T.A. Lebedkina and V.S. Gornakov. Materials Science Forum. 783-786, 204 (2014).

24. S. V. Makarov, V.A. Plotnikov, A.I. Potekaev. Russian Physics Journal. 54 (3), 314 (2011).

25. S. V. Makarov, V.A. Plotnikov, A.I. Potekaev. Russian Physics Journal. 57 (4), 436 (2014).

26. J. Robinson. International Materials Reviews. 39, 217 (1994).

27. Y. Estrin, L.P. Kubin. Materials Science and Engineering, A. 137,125 (1991).
28. M. M. Krishtal. The Physics of Metals and Metallography. 75 (5), 480 (1993).

29. M. M. Krishtal, D.L. Merson. The Physics of Metals and Metallography. 81 (1), 104 (1996).

30. A. A. Shibkov, A.E. Zolotov, M. F. Zheltov, M. F. Hasanov, A. A. Denisov. Physics of the Solid State. 56 (5), 848 (2014). (in Russian) [А.А. Шибков, А.Е. Золотов, М.Ф. Желтов, М.Ф. Гасанов, А. А. Денисов. ФТТ. 56 (5), 848 (2014).]

31. V.G. Badalyan, N.N. Vorontsov, V.F. Kazantsev et al. The Physics of Metals and Metallography. 54 (6), 1191 (1982). (in Russian) [В.Г. Бадалян, Н.Н. Воронцов, В. Ф. Казанцев и др. ФММ. 54 (6), 1191 (1982).]

32. V.F. Kazantsev, V.G. Badalyan. The Physics of Metals and Metallography. 55 (1), 191 (1983). (in Russian) [В. Ф. Казанцев, В. Г. Бадалян. ФММ. 55 (1), 191 (1983).]

33. S. A. Zhernov, I.F. Omelyanenko, A.F. Sirenko. The Physics of Metals and Metallography. 58 (3), 589 (1984). (in Russian) [C.А. Жернов, И.Ф. Омельяненко, А.Ф. Сиренко. ФММ. 58 (3), 589 (1984).]

34. L. Hausson, F. Tholen. Ultrasonics. March, 57 (1978).

35. V.I. Ushakov, I.F. Omelyanenko, A.F. Sirenko. The Physics of Metals and Metallography. 59 (5), 584 (1985). (in Russian) [В.И. Ушаков, И.Ф. Омельяненко, А. Ф. Сиренко. ФММ. 59 (5), 584 (1985).]

36. M.M. Myshlyaev, V. V., Shpeisman, V.V. Klubovich, M. M. Kulak, G. Liu. Physics of the Solid State. 57 (10), 1986 (2015). (in Russian) [M. М. Мышляев, В.В.Шпейзман, В. В. Клубович, М. М. Кулак, Г. Лю. ФТТ. 57 (10), 1986 (2015).]

37. S. V. Makarov, V.A. Plotnikov, A. I. Potekaev, L. S. Grinkevich. Russian Physics Journal. 57 (12), 1676 (2015).

38. S. V. Makarov, V.A. Plotnikov, A.I. Potekaev. Russian Physics Journal. 57 (7), 950 (2014).

39. E. V. Kozlov, L.E. Popov, M.D. Starostenkov. Russian Physics Journal. 15 (3), 107 (1972). (in Russian) [Э.В. Козлов, Л.Е. Попов, М.Д. Старостенков. Изв. ВУЗов. Физика. 15 (3), 107 (1972).]

40. B. F. Demyanov, A. S. Dragunov, A. V. Weckman. Bulletin of the Altai State University. 1/2 (65), 158 (2010). (in Russian) [Б. Ф. Демьянов, А. С. Драгунов, А. В. Векман. Известия Алтайского государственного университета. $1 / 2$ (65), 158 (2010)].

41. A. V. Weckman, B.F. Demyanov, A.S. Dragunov. The Physics of Metals and Metallography. 116 (6), 586 (2015).

42. F. A. Lindemann. Phys. Z. 11, 609 (1911).

43. M.N. Magomedov. Study of interatomic interaction, vacancy formation and self-diffusion in crystals. Moscow, Fizmatlit (2010) 544 p. (in Russian) [М. Н. Магомедов. Изучение межатомного взаимодействия, образования вакансий и самодиффузии в кристаллах. Москва, Физматлит (2010) 544 с.]

44. D.S. Sanditov. Journal of Experimental and Theoretical Physics. 142(1), 123 (2012). (in Russian) [Д.С. Сандитов. ЖЭТФ. 142(1), 123 (2012).]

45. E.E. Slyadnikov. Physics of the Solid State. 46(6), 1065 (2004). (in Russian) [Е.Е. Слядников. ФТТ. 46(6), 1065 (2004).]

46. O. Guerrero, M. Marucho. Journal of Materials Science and Engineering B. 3, 153 (2013). 